OPEN

SUBJECT AREAS:

COGNITIVE AGEING

COGNITIVE NEUROSCIENCE

Received

16 August 2013

Accepted

8 May 2014

Published

29 May 2014

Correspondence and requests for materials should be addressed to

I.-T.K. (iris.kolassa@ uni-ulm.de)

\section{Resting-state slow wave power, healthy aging and cognitive performance}

\author{
Eleni L. Vlahou', Franka Thurm ${ }^{2}$, Iris-Tatjana Kolassa ${ }^{1,3} \&$ Winfried Schlee ${ }^{1,3}$
}

${ }^{1}$ Clinical \& Biological Psychology, Institute of Psychology \& Education, University of Ulm, Germany, ${ }^{2}$ Department of Psychology, TU Dresden, Germany, ${ }^{3}$ Department of Psychology, University of Konstanz, Germany.

Cognitive functions and spontaneous neural activity show significant changes over the life-span, but the interrelations between age, cognition and resting-state brain oscillations are not well understood. Here, we assessed performance on the Trail Making Test and resting-state magnetoencephalographic (MEG) recordings from 53 healthy adults (18-89 years old) to investigate associations between age-dependent changes in spontaneous oscillatory activity and cognitive performance. Results show that healthy aging is accompanied by a marked and linear decrease of resting-state activity in the slow frequency range $(0.5-$ $6.5 \mathrm{~Hz}$ ). The effects of slow wave power on cognitive performance were expressed as interactions with age: For older ( $>54$ years), but not younger participants, enhanced delta and theta power in temporal and central regions was positively associated with perceptual speed and executive functioning. Consistent with previous work, these findings substantiate further the important role of slow wave oscillations in neurocognitive function during healthy aging.

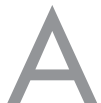

well-established finding from both cross-sectional and longitudinal comparisons is that during adulthood and aging, performance on numerous perceptual and cognitive functions shows a continuous and reliable decline ${ }^{1-4}$. This effect is even more pronounced in behavioral and neuropsychological tasks assessing speech perception, working memory, speed of processing, executive functions, reasoning and spatial orientation $^{1-6}$, although significant individual differences exist, with some high-performing older adults maintaining superior cognitive performance ${ }^{7}$.

Neurobiological explanations for age-related cognitive decline have focused on losses of grey and white matter volume $^{1,8}$, decreased dopaminergic neurotransmission during cognitively demanding tasks ${ }^{9}$, and reduction in hippocampal subregions, associated with impaired memory performance ${ }^{10,11}$. Other studies show that agedependent white matter alterations are associated with decrements in performance in tests assessing speed of processing, memory and executive functions $\mathrm{s}^{1,12}$.

Aging is also characterized by significant alterations in brain oscillatory activity ${ }^{13-24}$. Studies show that during physiological aging there is a generalized slowing of EEG activity, an increase in beta power, an amplitude decrease in lower alpha $(8-10.5 \mathrm{~Hz})$ and a slowing of peak alpha frequency ${ }^{13-17}$. A less consistent picture emerges for frequencies in the slow wave range. In some studies, healthy aging has been associated with increases in delta $(1-4 \mathrm{~Hz})$ and theta $(4-7.5 \mathrm{~Hz})$ power, whereas other studies have shown decreases ${ }^{14-23}$.

There is strong empirical support to the hypothesis that neural oscillations coordinate information transfer in the brain, support plasticity processes and subserve important perceptual and cognitive functions such as sensory feature binding, speech perception, memory and attention ${ }^{25-29}$. Of particular interest in the present study is the relation between spontaneous oscillatory activity, healthy aging and cognitive performance. Resting state recordings without any specific task are a useful tool to investigate the large-scale functional organization of the human brain and are used to study the default activity of functional brain networks ${ }^{30-32}$. Numerous studies have investigated possible links between age-related alterations in resting neural activity and cognitive performance. Examining 550 individuals between 11-70 years old, Clark et a ${ }^{13}$ showed that spontaneous frontal peak alpha frequency was positively associated with working memory performance, an effect independent of age. Decreases in magnitude of alpha sources during healthy aging have been associated with the functioning of the cholinergic basal forebrain system, affecting thalamo-cortical and cortico-cortical processing and resulting in reduced visuospatial attention ${ }^{17}$.

Recent work suggests that ongoing oscillatory activity in the slow frequency range $(<8 \mathrm{~Hz})$ is implicated in age-associated cognitive decline, but different patterns have been reported for healthy and pathological populations (e.g. ${ }^{18,19,23,33-36}$ ). For demented older adults, several studies show that enhanced delta and theta power predict neurocognitive impairment ${ }^{33,35,36}$. For example, Fernandez et $\mathrm{al}^{33}$ showed that patients suffering from Alzheimer's 
a

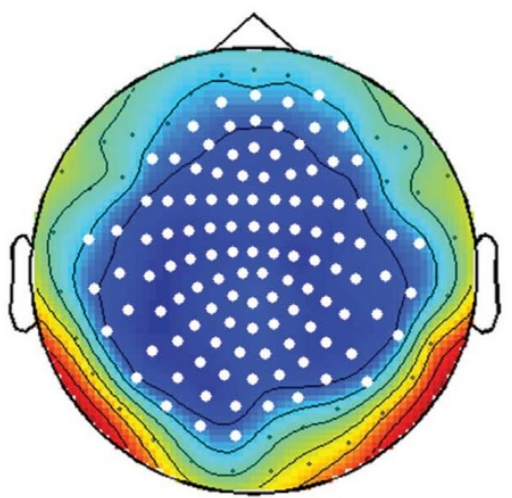

$P=.009$ b $\quad r=-.58$
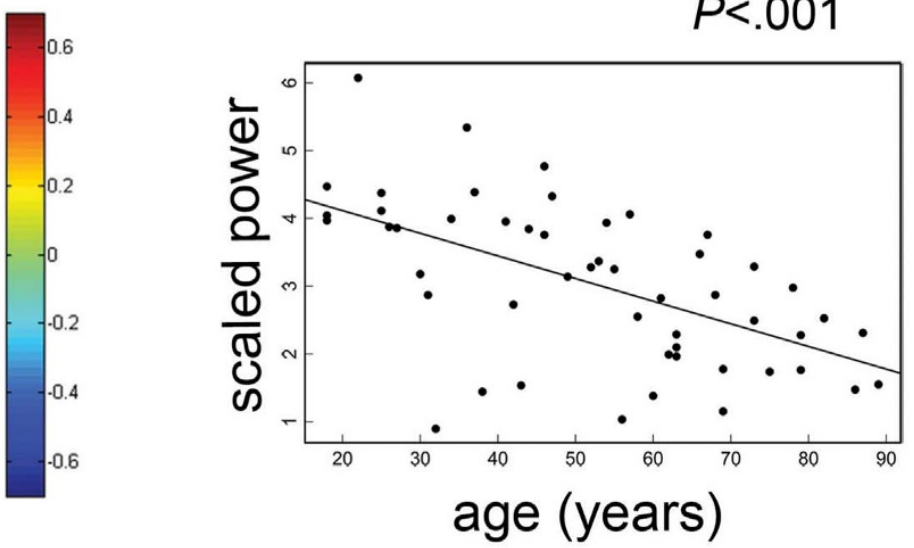

\section{$<7 \mathrm{~Hz}$}

Figure $1 \mid$ (a) Topographic representation of the scalp distribution of Pearson product moment correlations between age and MEG power for slow-waves $(0.5-6.5 \mathrm{~Hz})$. Blue regions indicate negative correlations, red regions indicate positive correlations. The cluster of sensors showing significant linear decrease in MEG power with age $(p=.009)$ is marked with white dots. (b) Scatterplot depicting linear correlations between cluster-averaged spectral power and chronological age $(\mathrm{r}=-.58, p<.001)$.

disease $(\mathrm{AD})$ had increased delta and theta activity in temporoparietal areas relative to controls, which was associated with deficient cognitive performance. Stomrud et $\mathrm{al}^{23}$ examined healthy elderly individuals over a period of 4.5 years. They showed that increases in posterior theta power correlated with decreases in mean peak frequency, cognitive speed impairment and changes in cerebrospinal fluid biomarkers which resembled $\mathrm{AD}$ pathology.

Other studies, however, report contrasting results: Examining 73 older adults, Finnigan and Robertson ${ }^{19}$ showed that spontaneous frontal and parietal theta power was positively associated with immediate and delayed verbal recall, attention, and executive functioning and suggested that in healthy older adults high resting theta power may be a neuromarker of successful neurocognitive aging. Cummins and Finnigan ${ }^{20}$ reported increased theta power for younger, compared to older adults during retention and recognition in a memory task, but no differences in task performance. Leirer et $\mathrm{al}^{18}$ found delta dipole density to decrease with physiological aging, but these alterations were not associated with neuropsychological tests assessing a broad range of cognitive abilities. Thus, despite recent interesting work, a clear characterization of the interrelations between healthy aging, cognitive performance and resting-state slow wave activity is not yet established.

The purpose of this study was twofold: Our first goal was to characterize the patterns of age-related alterations in resting-state power across the lifespan. Using magnetoencephalographic (MEG) recordings from 53 healthy adults (18-89 years old), we performed correlations between spectral power and age, using all frequencies (1$100 \mathrm{~Hz}$ ) and sensors across the head. A second motivation was to investigate the behavioral significance of these alterations, focusing on frequencies in the slow wave range. We used performance on the Trail Making Test ${ }^{37}$. Part A (TMT-A) is mostly associated with visual search and perceptual speed whereas part B (TMT-B) is mostly implicated in working memory and executive components ${ }^{38}$. We focused on neuropsychological tests that assess key cognitive functions which are primarily affected by age $e^{18,30,37}$. In addition, there is evidence that working memory, perceptual speed and cognitive control processes are associated with slow wave power ${ }^{23,27,39-43}$, and are thus expected to show age-differential spectral power alterations. Cognitive performance was regressed against age, slow frequency power and the interaction between the two.

\section{Results}

Cluster-based correlations between MEG power and age. Clusterbased permutations revealed a significant linear decrease of spontaneous oscillatory activity with increasing age for the slow frequency range of $0.5-6.5 \mathrm{~Hz}(p=.009)$. The decrease in slow wave power was widespread over large parts of the brain, affecting almost the entire sensor array (Figure 1a). This negative relation is illustrated in the scatterplot in Figure 1b: For each subject, we averaged power estimates from all frequency $\times$ sensor samples within the cluster and correlated these values with age. Pearson $r$ was $-.58(p<.001)$. In Figure 2, participants were divided into a "younger" (18-54 years of age, $\mathrm{n}=26$ ) and "older" (55-89 years of age, $n=27$ ) group, based on a median split. The graph shows reduced slow wave power for "older" compared to "younger" participants.

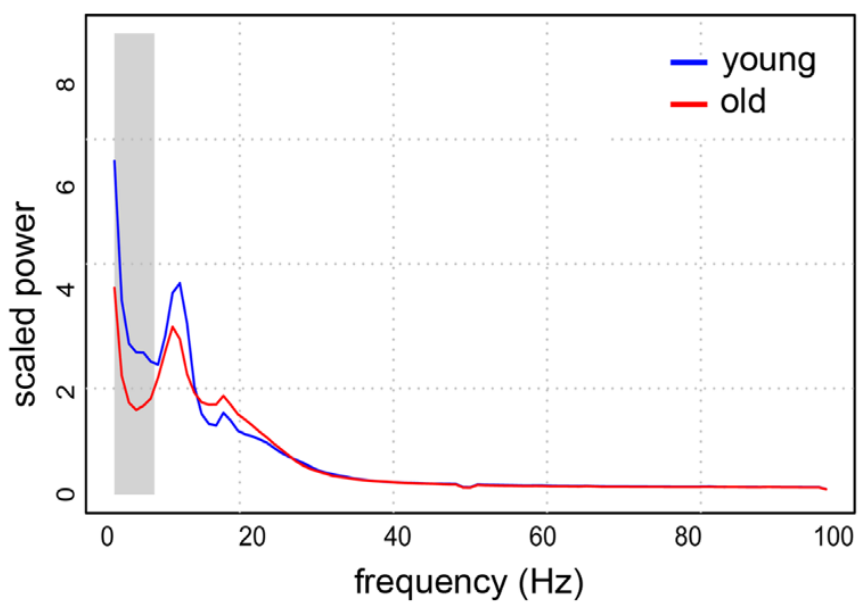

Figure $2 \mid$ Scaled spectral power averaged over all sensors from the slow wave cluster. Significant frequencies are shaded in gray. Participants were divided into a "younger" $(\mathrm{n}=26$, blue line $)$ and an "older" $(\mathrm{n}=27$, red line) group by median split. Older participants exhibit reduced spectral power compared to younger for slow wave frequencies. The small peak at $16.6 \mathrm{~Hz}$ represents technical noise resulting from a railway system that operates in a distance of approximately $1 \mathrm{~km}$ to the lab. 


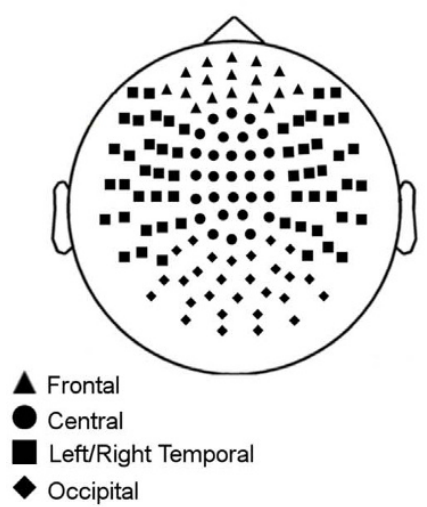

b

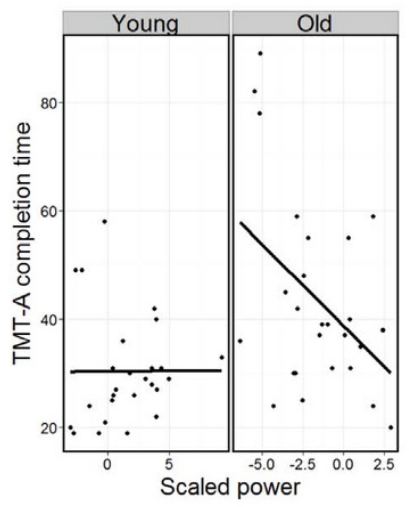

C

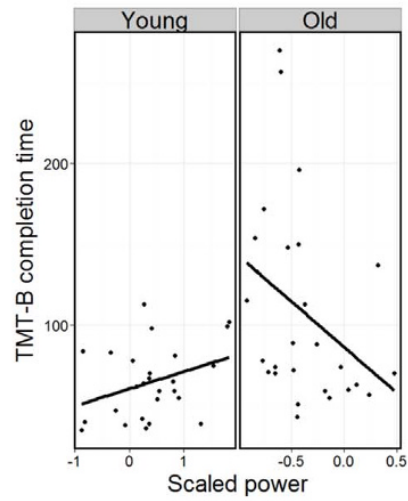

d

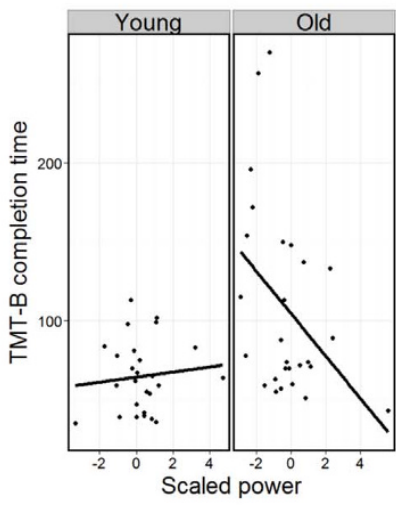

Figure $3 \mid$ (a) Illustration of the position of the selected sensor clusters for the five defined regions (Frontal, Central, Left Temporal, Right Temporal and Occipital). (b-d) Scatterplots depicting linear correlations between slow wave activity in temporal and central regions and performance (completion time) on the Trail Making Test, separately for "younger" $(\mathrm{n}=26)$ and "older" $(\mathrm{n}=27)$ participants (based on a median split). (b) Associations between performance on TMT-A and delta power $(0.5-4 \mathrm{~Hz})$ in the right temporal region. (c) Associations between performance on TMT-B and delta power in the central region. (d) Associations between performance on TMT-B and theta activity in the left temporal region.

No significant associations were observed between age and any other frequencies, although there was a non-significant trend for decreased gamma power over posterior sites $(p=.065)$.

Associations between age, theta power and individual peak alpha frequency (IAF). No significant associations were observed between IAF and age for frontal $(\beta=0.01, \mathrm{SE}=0.02, \mathrm{t}=0.75, p=.46)$, central $(\beta=0.01, \mathrm{SE}=0.02, \mathrm{t}=0.65, p=.52)$, occipital $(\beta=-0.02$, $\mathrm{SE}=0.01, \mathrm{t}=-1.59, p=.12)$ and left $(\beta=-0.03, \mathrm{SE}=0.02, \mathrm{t}=$ $-1.89, p=.06)$ and right $(\beta=-0.02, \mathrm{SE}=0.02, \mathrm{t}=-1.07, p=.29)$ temporal regions. Similarly, there were no associations between IAF and theta power for central $(\beta=-0.02, \mathrm{SE}=0.98, \mathrm{t}=-0.02, p$ $=.98)$, occipital $(\beta=-0.24, \mathrm{SE}=0.25, \mathrm{t}=-0.94, p=.35)$ and left $(\beta=-0.13, \mathrm{SE}=0.19, \mathrm{t}=-0.70, p=.49)$ and right $(\beta=-0.23, \mathrm{SE}$ $=0.12, \mathrm{t}=-1.89, p=.07)$ temporal regions. For the frontal region, $\mathrm{a}$ significant association was observed $(\beta=-0.77, \mathrm{SE}=0.26, \mathrm{t}=$ $-2.99, p=.004)$. However, Cook's distance and robust regression analyses identified three observations as outlying cases. After removal of the detected cases, no significant associations were observed $(\beta=-0.34, \mathrm{SE}=0.19, \mathrm{t}=-1.76, p=.09)$. In the following we report results based on unadjusted frequency bands.

Multiple regressions between aging, spectral amplitude and cognitive performance. We showed previously that in the current sample of healthy adults, age has a negative effect on performance in the Trail Making Test (TMT-A: $r=.51 ; p<.001$, TMT-B: $r=.55$, $\left.p<.001 ; \sec ^{30}\right)$. Initial exploratory correlations between spectral power and cognitive performance revealed different linear trends across different age groups. To further investigate this, we ran multiple linear regressions, using age, slow wave power and their interaction as predictors and performance on the Trail Making Test as the dependent variable. IAF was initially entered as a covariate but it did not reduce $\mathrm{AIC}^{46}$ by $\geq 2$, therefore the more parsimonious models were selected. Results from F-tests comparing the full with reduced models, excluding the effect under consideration, showed no significant contribution of IAF.

Our analyses revealed that the interaction between age and slow wave power at temporal and central regions was a significant predictor of performance in the Trail Making Test. The presence of a significant interaction suggests that the effects of power on performance are conditional on the participants' age. Figure 3 illustrates the nature of this relation: scatterplots are presented, depicting associa- tions between cognitive performance and spectral power separately for "younger" ( $<55$ years old, $\mathrm{n}=26)$ and "older" $(\mathrm{n}=27)$ participants (based on a median split).

In the delta frequency, significant interactions were observed for both TMT-A (right temporal region) and TMT-B (central region). Entering IAF as a covariate did not improve model fit. Specifically, for TMT-A in the right temporal region, AIC was 422.71 for the regression model including IAF and 422.71 for the reduced model (AIC difference $<.01$ ). For TMT-B in the central region, AIC was 547.47 for the full and 545.52 for the reduced model (AIC difference $=1.95)$. F-tests showed no significant contribution of IAF (TMT-A: $\mathrm{F}(1,48)=1.84, p=.18$; TMT-B: $\mathrm{F}(1,48)=0.04, p=.84)$, therefore the simpler models were retained.

For TMT-A the overall model accounted for $38 \%$ of the variance (adjusted $\left.\mathrm{R}^{2} ; \mathrm{F}(3,49)=11.73, p<.001\right)$. There was a significant interaction between Age $\times$ Power (Age $\times$ Power: $\beta=-0.09$, SE $=0.02, \mathrm{t}=-3.27, p=.002$; age: $\beta=0.31, \mathrm{SE}=0.09, \mathrm{t}=3.11, p=$ .003 ; power: $\beta=-1.44, \mathrm{SE}=0.65, \mathrm{t}=-2.22, p=.03$ ). Figure $3 \mathrm{~b}$ reveals a different pattern for different age groups: For older, but not younger participants, more power was associated with less completion time. For TMT-B, there was a significant interaction between delta power in central areas and age (adjusted $\mathrm{R}^{2}=.40, \mathrm{~F}(3,49)=$ 12.54, $p<.001$; interaction: $\beta=-1.73, \mathrm{SE}=0.51, \mathrm{t}=-3.38, p=$ .001 ; age: $\beta=1.18, \mathrm{SE}=0.33, \mathrm{t}=3.63, p<.001$; power: $\beta=-15.07$, $\mathrm{SE}=10.31, \mathrm{t}=-1.46, p=.15)$. Figure $3 \mathrm{c}$ shows a negative association between power and completion time for older participants.

In the theta frequency, the interaction between spectral power in the left temporal region and age was a significant predictor of performance on TMT-B. As before, adding IAF did not contribute to the model (AIC: 541.25 for the full and 539.73 for the reduced model; AIC difference $=1.51 ; \mathrm{F}(1,48)=0.44, p=.51)$ and was removed. The model accounted for $46 \%$ of the variance (adjusted $\mathrm{R}^{2} ; \mathrm{F}(3,49)=$ 15.87, $p<.001$; interaction: $\beta=-0.71, \mathrm{SE}=0.19, \mathrm{t}=-3.79, p<$ 0.001 ; age: $\beta=1.25, \mathrm{SE}=0.26, \mathrm{t}=4.74, p<.001$; power: $\beta=-6.12$, $\mathrm{SE}=3.15, \mathrm{t}=-1.94, p=0.06$ ). Figure $3 \mathrm{~d}$ shows a similar pattern: for older participants, increased slow wave power was associated with better performance.

In sum, our results suggest that the effects of slow wave power on cognitive performance were expressed as interactions with age: for older ( $>54$ years) participants, higher delta and theta power was associated with improved performance in the Trail Making Test. 


\section{Discussion}

Using MEG, this study aimed to investigate the effects of healthy aging on spontaneous spectral power in the $0.5-100 \mathrm{~Hz}$ frequency range and to explore associations between age-dependent power alterations and behavioral performance in the Trail Making Test.

Slow wave power $(<7 \mathrm{~Hz})$ linearly decreased with increasing age. No significant age-related changes were observed for any other frequency band. Spectral power decrements were widespread across the scalp, affecting almost the entire sensor array. These findings are in line with previous research, showing decreases in delta and theta power during physiological aging ${ }^{17,18,20}$. Moreover, the associations of spectral power with cognitive performance were expressed as interactions with age: for older participants, enhanced resting delta and theta power predicted improved neurocognitive function in tests assessing perceptual speed, working memory and cognitive control processes.

Studies focusing on pathological aging generally agree that enhanced slow wave spectral activity is associated with cognitive impairment (e.g. ${ }^{33,35,36}$ ). For example, Fernández et al. $^{33}$ reported enhanced theta/delta dipole density on $\mathrm{AD}$ patients compared to healthy controls, associated with deficient neurocognitive functioning. Grunwald and colleagues ${ }^{35,36}$ correlated theta power from seniors with different stages of cognitive impairment (forming a continuum from healthy to demented subjects) with hippocampal volume and found significant negative linear associations, suggesting that the observed slow wave alterations in the cortex may be accounted for by the neuronal loss in the hippocampus.

For healthy aging, reports are less consistent. Although it is well documented that it is accompanied by significant changes in slow wave activity, there is still much controversy over the specific patterns of these alterations, their underlying causes and their behavioral significance (e.g. ${ }^{15,18,19}$ ). Some studies report that age-related power increases in slow frequencies are indicative of subsequent cognitive deterioration. Prichep et al. ${ }^{34}$ showed that baseline excess of absolute and relative theta power could predict future cognitive decline in subjects who initially reported subjective cognitive complaints but showed no objective evidence of cognitive impairment. Stomrud et $\mathrm{al}^{23}$ reported that increased theta power in healthy older adults correlated with reductions in mean peak frequency, changes in cerebrospinal fluid and decreased cognitive speed. These alterations resembled $\mathrm{AD}$ pathology, therefore the authors interpreted their findings as reflecting possible early mild brain pathology, already present in healthy elderly individuals.

Other studies, however, show positive associations between slow wave activity and cognitive performance. Finnigan and Robertson ${ }^{19}$ examined healthy seniors ( $>56$ years old) and showed positive relations between resting theta and measures of immediate and delayed verbal recall, attention and executive function. A recent study ${ }^{41}$ showed that in healthy older adults, targeted cognitive training using a rich multimodal videogame induced increases in midline theta power and long-range theta coherence between frontal and posterior brain regions, significant improvement in the training regime and generalization of learning to untrained cognitive control skills.

In an attempt to account for the somewhat inconsistent findings between healthy and demented populations, Finnigan and Robertson ${ }^{19}$ suggested that for the latter, enhanced slow wave power may be related to alpha slowing and transitions of the peak resting frequency from alpha to theta, resulting in enhanced theta power. As noted by Klimesch ${ }^{14}$, parallel reductions in alpha and increases in theta power, which are often observed in older adults, may be related to age-related neurological disorders and not aging per se. However, when alpha slowing is not present, high theta power may be a marker of healthy neurocognitive function ${ }^{19}$. This conclusion is consistent with our findings. Here, we analyzed data from a population of healthy seniors, excluding carefully participants who presented evidence of neurological or other age-related pathologies. We did not observe significant associations between age, individual peak alpha frequency and theta power.

In sum, our results suggest that (a) healthy aging is accompanied by a widespread decrease in slow wave power and worse performance on various cognitive tests; this phenomenon could underlie decreased synaptic plasticity and communication between functional networks, associated with impaired cognitive function, (b) for healthy older adults who are able to maintain relatively higher levels of resting slow wave power, improved performance is observed in tests assessing perceptual speed, working memory and executive functioning. This conclusion is consistent with numerous studies which implicate slow wave oscillations in critical cognitive functions, including speech perception, working memory, perceptual decision making, executive functioning as well as in facilitating interaction and coordinating different brain regions into functional networks ${ }^{27,40-45}$. It is also in line with recent proposals which consider slow wave activity as a neural correlate of enhanced control engagement, structuring and integrating the dynamics of neuronal communication in order to effectively adapt to current behavioral demands ${ }^{42}$ and which can be used to predict individuals who are more able to benefit from cognitive training regimes and show broad-based transfer effects to untrained cognitive control tasks ${ }^{40,41}$.

\section{Methods}

Participants. Fifty-three healthy volunteers ( 23 men, 30 women), between 18 to 89 years of age (mean age 53.1 years, $S D=20.1$ ) participated in this study. They were recruited via advertisements in the local newspaper and radio station and via flyers posted at the University of Konstanz, in residential homes for older adults and senior citizen centers. All participants gave written informed consent prior to the diagnostic interview, neuropsychological testing and MEG recordings and received 30 Euro as compensation. Exclusion criteria were dementia or probable dementia according to DSM-IV-TR ${ }^{47}$, psychiatric disorders, psychopharmacological medication, history of severe head injuries or neurological problems, left-handedness and metal objects in the body. The study was approved by the ethics committee of the University of Konstanz and the methods were carried out in accordance with the approved guidelines.

Neuropsychological assessment. Prior to the MEG recordings, all participants were screened with the Mini International Neuropsychiatric Interview (M.I.N.I. ${ }^{48}$ ). Handedness was assessed using the Edinburgh Handedness Inventory ${ }^{49}$. Cognitive performance was assessed using with a variety of tests (for a complete description $\mathrm{se}^{18,30}$ ) for a total duration of approximately 2 hours. In the current study we used Trail Making Test A (TMT-A; range, 0-180 seconds) and B (TMT-B; range, 0-300 seconds) from the Consortium to Establish a Registry for Alzheimer's Disease (CERAD)-NP-plus neuropsychological test battery ${ }^{50}$.

MEG recordings and analysis. Neuromagnetic data were recorded with a 148 channel whole-head magnetometer system (MAGNES TM $2500 \mathrm{WH}, 4 \mathrm{D}$ Neuroimaging, San Diego, CA, USA), installed in a magnetically shielded and quiet room (Vacuumschmelze, Hanau, Germany). The recording duration was set to 5 minutes. During this period participants were laying in a comfortable supine position awake and with their eyes open. They were required to fixate on a mark on the ceiling. Furthermore, they were instructed not to move their eyes and body and to avoid engaging in any specific mental activity. To ensure compliance with the experimental requirements, their behavior was monitored with a camera installed inside the chamber.

Continuous data were recorded with a hard-wired high-pass filter of $0.1 \mathrm{~Hz}$ and a sampling rate of $678.17 \mathrm{~Hz}$. MEG data were analyzed with the fieldtrip toolbox ${ }^{51}$ (www.ru.nl/fcdonders/fieldtrip), run in a Matlab environment (www.mathworks. com). All data sets were down-sampled to $600 \mathrm{~Hz}$ and cut into epochs of $2 \mathrm{~s}$. Epochs containing eye movements, eye blinks or muscle artifacts were detected and excluded from further analysis based on visual inspection. Next, an independent component analysis (ICA) was calculated for each individual data set to identify and reject the components that reflect the heart beat ('fastica' algorithm, implemented in $\mathrm{EEGLAB}^{52}$ ). After artifact correction, 90 trials ( $180 \mathrm{~s}$ in total) were randomly selected from the remaining trials and used for subsequent analyses. Prior to power spectra calculations, the MEG data from each participant were realigned to a standard sensor position with respect to the participant's head ${ }^{53}$.

Frequency spectral analysis. For each subject, the realigned MEG data were transformed into individual frequency power spectra by applying a Fast Fourier Transformation (FFT) to $2000 \mathrm{~ms}$ data epochs. The output of this procedure was the mean (averaged over the 90 trials) power for each frequency bin in the range of 0.5$100 \mathrm{~Hz}$ (with a $0.5 \mathrm{~Hz}$ frequency resolution, resulting in 200 frequency bins), for each of the 148 channels. Power values were normalized by calculating the mean power from all sensors and frequencies for each individual subject. Subsequently, each 
separate sample (frequency $\times$ sensor power estimate) was divided by the subject's mean power.

Correlations between spectral power and age. Our first goal was to investigate alterations of spontaneous MEG power during healthy aging, examining the full frequency spectrum across the whole head. In order to perform correlational analyses between power and age while effectively controlling the type I error in a situation involving multiple comparisons (power values from 53 participants, 148 channels and 100 frequencies), we used a non-parametric cluster-based permutation procedure $^{54}$, implemented in Fieldtrip ${ }^{51}$.

First, all frequency $\times$ channel samples were correlated with age. Samples that survived the initial test (i.e., the uncorrected p-value was less than .01) were clustered based on spatio-spectral proximity. Clustering was performed separately for samples showing positive and negative associations with age. An additional constraint was that within each cluster, each sensor had at least three neighboring sensors. Clusterlevel statistics were obtained by summing the frequency $\times$ channel sample statistics within each cluster. The maximum of these was used to test the significance of our results against a randomization distribution. This distribution was obtained by randomly permuting the original data, taking the maximum cluster-level statistic and repeating this process for 1000 times. The probability of obtaining a statistic from this distribution larger than our actual cluster statistic was tested.

Associations between age, spectral power and cognitive performance. To investigate the interrelations between healthy aging, MEG power and cognitive performance, we ran multiple linear regressions using R (R Core Development Team, R Foundation for Statistical Computing, Vienna). We used spectral power, age and their interaction as predictors, and performance on neuropsychological tests as the dependent variable. Individual peak alpha frequency (IAF; defined as the frequency with the largest power in the range of $8-13 \mathrm{~Hz}$ ) was entered as a covariate.

Spectral power estimates for each participant were calculated in the following way: MEG sensors were grouped into different regions, corresponding to five major brain areas: frontal (17 sensors), central (29), occipital (29) and left and right temporal (28 each; Figure 3a). For each participant, spectral power was averaged over all sensors within each region, separately for the delta $(0.5-4 \mathrm{~Hz})$ and theta $(4.5-7.5 \mathrm{~Hz})$ frequency bands. This process resulted in a 53 (participants) $\times 2$ (frequencies) $\times 5$ (region) matrix of power estimates which were entered in subsequent regression analyses.

For cognitive tests we used completion time on Trail Making Test A and B. Performance on these tests has been associated with key cognitive functions, including cognitive control processes, working memory and perceptual speed ${ }^{37,38}$. All these measures have been shown to be negatively affected by age $e^{1,13,23,30}$ and to be modulated by neural activity in the delta and theta range $27,40-44$. Thus, of particular interest in the current study was to explore the associations between slow wave spectral power and performance on these tests across the life-span.

Separate tests were performed for each frequency, region and test, yielding a total of 20 tests. $P$ values were adjusted using Holm's stepwise procedure ${ }^{55}$. Both predictors were mean-centered, to reduce multicollinearity and to facilitate interpretation of coefficients. Variance Inflation Factors were low (all $<2$ ), suggesting that results were not biased by multicollinearity. To test the reliability of the model in the presence of outliers and heteroscedasticity, whenever results were significant we performed additional robust regressions (with iteratively re-weighted least squares using the "Imrob" function from "robustbase" package in the R environment). We report results from ordinary least-squares regression which remained significant using robust regressions.

Associations between age, theta power and IAF. For each region, additional regressions were performed between (a) IAF and age, and (b) IAF and theta power. Results are reported above.

1. Hedden, T. \& Gabrieli, J. D. E. Insights into the ageing mind: a view from cognitive neuroscience. Nat Rev Neurosci 5, 87-96 (2004).

2. Salthouse, T. A. Selective review of cognitive aging. J Int Neuropsychol Soc 16, 754-760 (2010).

3. Maddox, T. W., Chandrasekaran, B., Smayda, K. \& Yi, H.-G. Dual systems of speech category learning across the lifespan. Psychol Aging 28, 1042-1056 (2013).

4. Adank, P. \& Janse, E. Comprehension of a novel accent by young and older listeners. Psychol Aging 25, 736-740 (2010).

5. Salthouse, T. A. When does age-related cognitive decline begin? Neurobiol Aging 30, 507-514 (2009).

6. Schaie, K. W., Willis, S. L. \& Caskie, G. I. The Seattle longitudinal study: relationship between personality and cognition. Aging, Neuropsychol C 11, 304-324 (2004).

7. Nyberg, L., Lövdén, M., Riklund, K., Lindenberger, U. \& Bäckman, L. Memory aging and brain maintenance. Trends Cogn Sci 16, 292-305 (2012).

8. Bartzokis, G. et al. Age-related changes in frontal and temporal lobe volumes in men: a magnetic resonance imaging study. Arch Gen Psychiatry 58, 461-465 (2001).

9. Bäckman, L., Lindenberger, U., Li, S.-C. \& Nyberg, L. Linking cognitive aging to alterations in dopamine neurotransmitter functioning: Recent data and future avenues. Neurosci Biobehav R 34, 670-677 (2010).
10. Shing, Y. L. et al. Hippocampal subfield volumes: age, vascular risk, and correlation with associative memory. Front Aging Neurosci 3, (2011).

11. Small, S. A., Schobel, S. A., Buxton, R. B., Witter, M. P. \& Barnes, C. A. A pathophysiological framework of hippocampal dysfunction in ageing and disease. Nat Rev Neurosci 12, 585-601 (2011).

12. Salthouse, T. A. Neuroanatomical substrates of age-related cognitive decline. Psychol Bul 135, 753-784 (2011).

13. Clark, C. et al. Spontaneous alpha peak frequency predicts working memory performance across the age span. Int J Psychophysiol 53, 1-9 (2004).

14. Klimesch, W. EEG alpha and theta oscillations reflect cognitive and memory performance: a review and analysis. Brain Res Rev 29, 169-195 (1999).

15. Rossini, P. M., Rossi, S., Babiloni, C. \& Polich, J. Clinical neurophysiology of aging brain: From normal aging to neurodegeneration. Prog Neurobiol 83, 375-400 (2007).

16. McEvoy, L. K., Pellouchoud, E., Smith, M. E. \& Gevins, A. Neurophysiological signals of working memory in healthy aging. Cognitive Brain Res 11, 363-376 (2001).

17. Babiloni, C. et al. Sources of cortical rhythms in adults during physiological aging: a multicentric EEG study. Hum Brain Mapp 27, 162-172 (2006).

18. Leirer, V. M. et al. Changes in cortical slow wave activity in healthy aging. Brain Imag Behav 5, 222-228 (2011).

19. Finnigan, S. \& Robertson, I. H. Resting EEG theta power correlates with cognitive performance in healthy older adults. Psychophysiol 48, 1083-1087 (2011).

20. Cummins, T. D. \& Finnigan, S. Theta power is reduced in cognitive aging. Int J Psychophysiol 66, 10-17 (2007).

21. Hartikainen, P., Soininen, H., Partanen, J., Helkala, E. L. \& Riekkinen, P. Aging and spectral analysis of EEG in normal subjects: a link to memory and CSF AChE. Acta Neurol Scand 86, 148-55 (1992).

22. Ho, M.-C. et al. Task-related brain oscillations in normal aging. Health 4, 762-768 (2012).

23. Stomrud, E. et al. Slowing of EEG correlates with CSF biomarkers and reduced cognitive speed in elderly with normal cognition over 4 years. Neurobiol Aging 31, 215-223 (2008)

24. Puligheddu. et al. Age distribution of MEG spontaneous theta activity in healthy subjects. Brain Topogr 17, 165-175 (2005).

25. Ward, L. M. Synchronous neural oscillations and cognitive processes. Trends Cogn Sci 7, 553-559 (2003).

26. Engel, A. K. \& Fries, P. Beta-band oscillations - signalling the status quo? Curr Opin Neurobiol 20, 156-165 (2010).

27. Kahana, M. J., Seelig, D. \& Madsen, J. R. Theta returns. Curr Opin Neurobiol 11, 739-744 (2001).

28. Bosseler, A. N. et al. Theta brain rhythms index perceptual narrowing in infant speech perception. Front Psychol 4, doi: 10.3389/fpsyg.2013.00690 (2013).

29. Giraud, A. L. \& Poeppel, D. Cortical oscillations and speech processing: emerging computational principles and operations. Nat Neurosci, 15, 511-517 (2012).

30. Schlee, W. et al. Development of large-scale functional networks over the lifespan. Neurobiol Aging 33, 2411-2421 (2012).

31. Greicius, M. D., Krasnow, B., Reiss, A. L. \& Menon, V. Functional connectivity in the resting brain: a network analysis of the default mode hypothesis. PNAS 100, 253-258 (2003)

32. Raichle, M. E. et al. A default mode of brain function. PNAS 98, 676-682 (2001)

33. Fernández, A. et al. Focal temporoparietal slow activity in Alzheimer's Disease revealed by magnetencephalography. Biol Psychiatry 52, 764-770 (2002).

34. Prichep, L. S. et al. Prediction of longitudinal cognitive decline in normal elderly with subjective complaints using electrophysiological imaging. Neurobiol Aging 27, 471-481 (2006)

35. Grunwald, M. et al. Correlation between cortical $\theta$ activity and hippocampal volumes in health, mild cognitive impairment, and mild dementia. J Clin Neurophysiol 18, 178-184 (2001).

36. Grunwald, M., Hensel, A., Wolf, H., Weiss, T. \& Gertz, H.-J. Does the hippocampal atrophy correlate with the cortical theta power in elderly subjects with a range of cognitive impairment? J Clin Neurophysiol 24, 22-26 (2007).

37. Tombaugh, T. N. Trail Making Test A and B: Normative data stratified by age and education. Arch Clin Neuropsychol 19, 203-214 (2004).

38. Sánchez-Cubillo, I. et al. Construct validity of the Trail Making Test: Role of taskswitching, working memory, inhibition/interference control, and visuomotor abilities. J Int Neuropsych Soc 15, 438-450 (2009).

39. Kouijzer, M. E. J., de Moor, J. M. H., Gerrits, B. J. L., Congedo, M. \& van Schie, H. T. Neurofeedback improves executive functioning in children with autism spectrum disorders. Res Autism Spect Dis 3, 145-162 (2009).

40. Mathewson, K. E. et al. Different slopes for different folks: Alpha and delta EEG power predict subsequent video game learning rate and improvements in cognitive control tasks. Psychophysiology 49, 1558-1570 (2012).

41. Anguera, J. A. et al. Video game training enhances cognitive control in older adults. Nature 501, 97-101 (2013).

42. Nigbur, R., Ivanova, G. \& Stürmer, B. Theta power as a marker for cognitive interference. Clin Neurophysiol 122, 2185-2194 (2011).

43. Raghavachari, S. et al. Gating of human theta oscillations by a working memory task. J Neurosci 21, 3175-3183 (2001).

44. Caplan, J. B. et al. Human $\theta$ oscillations related to sensorimotor integration and spatial learning. J Neurosci 23, 4726-4736 (2003). 
45. Anderson, K. L., Rajagovindan, R., Ghacibeh, G. A., Meador, K. J. \& Ding, M. Theta oscillations mediate interaction between prefrontal cortex and medial temporal lobe in human memory. Cereb Cortex 20, 1604-1612 (2010).

46. Akaike, H. [Information theory as an extension of the maximum likelihood principle] Second International Symposium on Information Theory [Petrov, B. N. \& Csaki, F. (eds.)] [267-281] (Akademiai Kiado, Budapest, 1973).

47. American Psychiatric Association: Diagnostic and Statistical Manual of Mental Disorders, Fourth Edition, Text Revision (DSM-IV-TR) (Washington, DC, 2000).

48. Ackenheil, M., Stotz, G., Dietz-Bauer, R. \& Vossen, A. I. Mini International Neuropsychiatric Interview. German version 5.0.0. (Munich, 1999).

49. Oldfield, R. C. The assessment and analysis of handedness: the Edinburgh inventory. Neuropsychologia 9, 97-113 (1971).

50. Morris, J. C., Mohs, R. C., Rogers, H., Fillenbaum, G. \& Heyman, A. Consortium to establish a registry for Alzheimer's disease (CERAD) clinical and neuropsychological assessment of Alzheimer's disease. Psychopharmacol Bull 24, 641-652 (1988).

51. Oostenveld, R., Fries, P., Maris, E. \& Schoffelen, J. M. FieldTrip: open source software for advanced analysis of MEG, EEG, and invasive electrophysiological data. Comput Intell Neurosci (2011).

52. Delorme, A. \& Makeig, S. EEGLAB: an open source toolbox for analysis of singletrial EEG dynamics including independent component analysis. J Neurosci Meth 134, 9-21 (2004)

53. Knösche, T. R. Transformation of whole-head MEG recordings between different sensor positions. Biomedizinische Technik 47, 59-62 (2002).

54. Maris, E. \& Oostenveld, R. Nonparametric statistical testing of EEG and MEGdata. J Neurosci Meth 164, 177-190 (2007)

55. Holm, S. A simple sequentially rejective multiple test procedure. Scand J Stat 6 , 65-70 (1979).

\section{Acknowledgments}

This work was supported by the Program DAAD/IKYDA 2012 (Program for the promotion of the exchange and scientific cooperation between Greece and Germany) and the WIN project of the Heidelberg Academy of Sciences awarded to ITK. We would like to thank Vera Leirer for her important contributions in data collection.

\section{Author contributions}

I.T.K. and E.V. designed the study. V.L. collected the data together with I.T.K.E.V. and W.S performed the MEG and statistical analyses. E.V. drafted the manuscript. E.V., I.T.K., F.T. and W.S. interpreted the results and revised the manuscript. I.T.K. provided funding for this study.

\section{Additional information}

Competing financial interests: The authors declare no competing financial interests.

How to cite this article: Vlahou, E.L., Thurm, F., Kolassa, I.-T. \& Schlee, W. Resting-state slow wave power, healthy aging and cognitive performance. Sci. Rep. 4, 5101; DOI:10.1038/ srep05101 (2014).

This work is licensed under a Creative Commons Attribution-NonCommercialShareAlike 3.0 Unported License. The images in this article are included in the article's Creative Commons license, unless indicated otherwise in the image credit; if the image is not included under the Creative Commons license, users will need to obtain permission from the license holder in order to reproduce the image. To view a copy of this license, visit http://creativecommons.org/licenses/by-nc-sa/3.0/ 